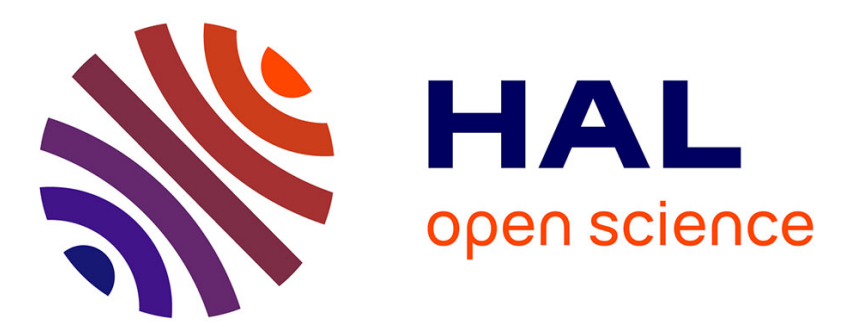

\title{
First principles determination of structural, electronic and lattice dynamical properties of a model dipeptide molecular crystal
}

\author{
Paul Robert Tulip, Simon Bates
}

\section{To cite this version:}

Paul Robert Tulip, Simon Bates. First principles determination of structural, electronic and lattice dynamical properties of a model dipeptide molecular crystal. Molecular Physics, 2009, 107 (20), pp.2201-2212. 10.1080/00268970903224955 . hal-00521569

\section{HAL Id: hal-00521569 \\ https://hal.science/hal-00521569}

Submitted on 28 Sep 2010

HAL is a multi-disciplinary open access archive for the deposit and dissemination of scientific research documents, whether they are published or not. The documents may come from teaching and research institutions in France or abroad, or from public or private research centers.
L'archive ouverte pluridisciplinaire HAL, est destinée au dépôt et à la diffusion de documents scientifiques de niveau recherche, publiés ou non, émanant des établissements d'enseignement et de recherche français ou étrangers, des laboratoires publics ou privés. 


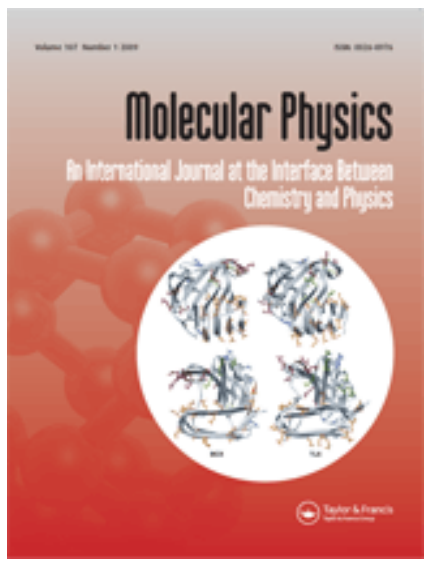

First principles determination of structural, electronic and lattice dynamical properties of a model dipeptide molecular crystal

\begin{tabular}{|r|l|}
\hline Journal: & Molecular Physics \\
\hline Manuscript ID: & TMPH-2009-0187.R1 \\
\hline Manuscript Type: & Full Paper \\
\hline Author: & 14-Jul-2009 \\
\hline Komplete List of Authors: & $\begin{array}{l}\text { Tulip, Paul; University of Edinburgh, School of Physics and } \\
\text { Astronomy } \\
\text { Bates, Simon; University of Edinburgh, School of Physics and } \\
\text { Astronomy }\end{array}$ \\
\hline \multicolumn{2}{|c|}{$\begin{array}{l}\text { ab initio, density functional theory, molecular crystals, lattice } \\
\text { dynamics }\end{array}$} \\
\hline $\begin{array}{l}\text { Note: The following files were submitted by the author for peer review, but cannot be converted } \\
\text { to PDF. You must view these files (e.g. movies) online. }\end{array}$ \\
\hline tulip.zip \\
\hline
\end{tabular}

\section{(1) ScholaroNE \\ Manuscript Central}




\title{
1. Introduction
}

\begin{abstract}
We present the results of $a b$ initio calculations of the structural, electronic and lattice dynamical properties of the solid-state crystal of the glycyl-l-alanine dipeptide. Intramolecular bond lengths are found to be in good agreement with experimental values; lattice constants are in reasonable agreement, although it is found that discprepancies do exist. A hierarchy of hydrogen bond strengths is found, with those between (oppositely-charged) amine and carboxy functional groups being strongest. The crystal is found to be an indirect-bandgap material, with indirect bandgaps $\approx 4.95 \mathrm{eV}$, compared to a direct bandgap of $5.00 \mathrm{eV}$. Analysis of the electronic structure reveals that the electronic states in the near vicinity of the energy gap arise from carboxylate and amide oxygen atoms. The arrangement of both molecules and hydrogen bonds in the unit cell is found to manifest itself in increased bandwidth along specific reciprocal space directions, reflecting coupling brought about by hydrogen bonds. Determination of the zone-centre lattice dynamical behaviour permits the IR absorption spectrum to be explained. Intermolecular hydrogen bonds are found to couple intramolecular motions in adjacent moelcules, revealing the importance of an accurate treatment of intermolecular interactions, even for high-frequency vibronic modes.
\end{abstract}

Keywords: ab initio; density functional theory; molecular crystals; lattice dynamics

Recent years have witnessed sustained and intense research efforts into proteins and peptides, using a combination of experimental [1-3], theoretical [4-6] and computational methods [7-10]. There are several reasons for this interest: firstly, peptides, as the building blocks of proteins are of fundamental biological importance - successfully understanding the biological physics of these molecules will provide a major advance in our biological understanding. Secondly, misfolding and aggregation of peptides and proteins has been implicated as being of critical importance in understanding the pathologies of diseases such as Alzheimer's and Creutzfeldt-Jakob's $[11,12]$. Promising potential treatment strategies for these diseases involve the development of peptidic inhibitors that may prevent, or reverse, aggregation [13-15].

There are, in addition, technological imperatives for this interest: hybrid bionanoparticle materials offer the promise of novel technological applications. For example, conjugation of peptides with metallic nano-clusters results in modification of optical, electronic and conformational properties [16-20]; peptide adsorption on metallic and mineral surfaces, meanwhile, is of relevance to the development of

*Corresponding author. Email: ptulip@ph.ed.ac.uk 
biosensors and bioarrays [21-24], whilst peptide-inorganic interfaces offer novel means of materials synthesis and nanoscale fabrication [25-28].

The common theme underpinning these disparate research avenues is the desire to develop, at the molecular level, a detailed and accurate description of the intermolecular interactions responsible for these phenomena and to thereby elucidate the relationship between peptide structure and function. Computational modelling is an invaluable tool that may be used to effect this goal; valuable insights have been gained using a variety of modelling techniques, as examination of the relevant literature reveals [29-34].

One of the most powerful elements of the computational physicist's armoury is provided by quantum mechanical electronic structure calculations. The power of these methods is intimately related to the realisation that the key to understanding the properties of any condensed matter system lies within the electronic structure: once the ground-state electronic structure has been accurately determined, this allows, in principle, a complete understanding of its physical properties. The Hohenberg-Kohn-Sham density functional theory (DFT) [35, 36] represents the state-of-the-art for such calculations. A long history of successful applications to a wide range of systems [37-41] attests to the power and flexibility of the method; and indeed, it is sufficiently powerful that it may be used as a predictive tool in its own right, rather than merely serving as a tool to confirm experimental results.

In this paper we make use of DFT calculations to investigate and elucidate the structural, electronic, dielectric and lattice dynamical properties of the solid state phase of the dipeptide glycyl-l-alanine. This dipeptide has recently been the subject of computational and experimental work: the gas-phase structure and vibrational properties have been determined [42], whilst some attempt has been made to extend this work to incorporate the condensed phase through a dielectric continuum treatment of solvent effects $[42,43]$. The hydration structure of the peptide in water has been studied using both classical and $a b$ initio molecular dynamics methods [44, 45]. More recently, the solution structure of the peptide in water at a 1:20 solute-solvent concentration has been investigated using neutron diffraction methods [46], whilst a recent study by the current authors [47] has addressed the nature of peptide aggregation in this system and elucidated the solvent structure using classical molecular dynamics simulations. The latter work has provided the stimulus for the current study: molecular crystals provide a well-defined system that may be analysed at a high level of theoretical treatment; in particular, the electronic structure itself can be investigated in some depth, allowing the nature of the intermolecular interactions to be elucidated. To the best of our knowledge, this is the first such systematic investigation of this dipeptide's solid state properties; previous investigations have concerned specific topics such as NMR shielding tensors [48, 49]. Indeed, there are relatively few works analysing the electronic structure of biomolecular crystals in depth; however, meaningful exploitation of such systems demands that the electronic structure be understood in detail, and the relationship to the crystal and molecular structure be understood. The objective of this work is to provide such a characterisation for this particular peptide.

The paper is structured as follows: in the next section we provide an overview of the theoretical and computational methods employed in this study, whilst in section 3 we discuss the structural properties. In section 4, we address the electronic structure. In section 5 we focus our attention upon the lattice dynamical and dielectric properties; finally, we conclude in section 6 . 


\section{Computational Approach}

We carry out our calculations within the plane wave pseudopotential (PWP) formalism of the Hohenberg-Kohn formulation of density functional theory (DFT) $[35,36]$. This is a powerful technique for the accurate computation of ground state properties. Despite the existence of the well-known bandgap problem [50], i.e. that DFT commonly estimates incorrect bandgaps, the qualitative topology of bandstructures obtained using DFT is generally considered to be correct, and we are therefore justified in using this concept to understand the electronic structure of the systems under consideration. The calculations are performed using the CASTEP [51] code, using norm-conserving pseudopotentials of the Kleinman-Bylander [52] form. The valence electron wavefunctions are expanded in a plane-wave basis set, with a kinetic energy cut-off of $1000 \mathrm{eV}$. Reciprocal space integrations have been carried out using a $3 \times 3 \times 3$ Monkhorst-Pack grid [53]; this yields eight symmetryreduced k-points, and converges total energies to better than $1 \mathrm{meV} /$ atom, a degree of accuracy comparable to that with regard to kinetic energy cut-off. Exchange and correlation are treated using the well-known generalised gradient approximation (GGA) due to Perdew and Wang [54]. This is known to be capable of accurately treating hydrogen-bonded molecular systems $[55,56]$, although it should be acknowledged that such semi-local functionals are intrinsically incapable of treating van der Waals interactions.

Our calculations fall into several distinct stages: firstly we determine the geometrical structures adopted at equilibrium by minimising the forces acting on nuclei using the well-known Hellmann-Feynman theorem. This involves optimisation of all atomic coordinates, in addition to allowing the unit cell to relax. We take experimentally determined structures [57] as our initial atomic positions. This approach amounts to identifying the minimum in the lattice energy. A large body of research into crystal structure prediction [58-60] indicates that such minimisation of the intermolecular potential energy represents a successful method for optimising crystal packing, and that density functional theory may be used to quantify such interactions, either directly, or through a DFT-derived forcefield. Once we have optimised these structures, we then solve the single-particle Kohn-Sham equations at a series of reciprocal space points to determine the eigenvalue spectrum.

Having determined the structural and electronic properties, we determine the lattice dynamical and dielectric properties of the crystal using the variational formalism of density functional perturbation theory (DFPT) of Gonze and Lee [61], as implemented within the CASTEP code [51, 62]. DFPT is a powerful method capable of determining the lattice dynamical properties of the crystal via a harmonic treatment; as such, it is incapable of treating anharmonicities. However, it has been successfully applied to such organic molecular systems [56, 62-64], which justifies our confidence in the accuracy of the assignments presented here.

In comparison to frozen-phonon approaches, the DFPT approach to lattice dynamics possesses the advantage that the zone-centre excitations may be readily accessed; further, that because it is formulated at the linear response level, it is possible to compute the system response to a combined perturbation (e.g. a lattice perturbation and an applied field). This allows the macroscopic electric fields induced by long-wave atomic displacements to be naturally incorporated within the calculation. These fields lead to the so-called "LO-TO splitting" [65] of zonecentre modes. Successful treatment of this phenomenon is essential for an accurate description of the normal modes.

In this work, we concern ourselves solely with the determination of the vibra- 
tional excitation spectrum at the Brillouin zone centre, as opposed to determining a full dispersion curve. The zone-centre phonons are of physical importance in that it is these modes that couple to electromagnetic radiation. Further, it should be noted that, whilst typically, DFPT is capable of describing vibrational frequencies to within $50 \mathrm{~cm}^{-1}$, molecular crystals may prove to be demanding tests of the theory. In particular, the soft potential surfaces of such systems ensure that extremely stringent (and computationally demanding) convergence criteria must be employed to determine low-lying intermolecular modes accurately [66]. For this reason we focus our attention instead upon the intramolecular modes, which are less demanding to describe adequately.

It is convenient at this stage to consider the molecular structure and labelling scheme employed in this study. In Figure 1 the zwitterionic structure of the glycyl-lalanine molecule is illustrated, along with the numbering convention used throughout.

\section{Structural Properties}

The initial crystal structure for the geometry optimisation was taken from the Xray diffraction data of Wang and Paul [57] at room temperature. These data suggest that the crystal forms an orthorhombic structure, with space group $P_{212121}$, with four molecules per unit cell. In Figure 2 we present this structure. It can be seen that, if the structure is viewed along the $a$-axis, then the molecules adopt a herring-bone-type-layering structure, in which layers "stack" along the $c$ axis. This maximises inter-molecular hydrogen bonding contacts. Our geometry optimisation calculations relax all internal degrees of freedom in addition to the unit cell parameters whilst maintaining a fixed system symmetry. In Table 1 the optimised unit cell parameters are listed. It can be seen that these are in good agreement with those obtained experimentally for both $\mathrm{XC}$ functionals employed (of the order of $2 \%$ ). In the $b$ and $c$ directions, the optimised lattice constants are longer than the experimental values, which is consistent with the known tendency of the GGA to underestimate binding $[41,50]$. In contrast, the $a$-axis is underestimated relative to experiment, suggesting instead a tendency to over-bind in this case. The experimental cell volume is $695.79 \mathrm{~A}^{3}$. The PW91 and PBE functionals predict larger cell volumes at $716.60 \mathrm{~A}^{\circ}$ and $719.88 \mathrm{~A}^{\circ}$, respectively. It should be noted that these calculations result in residual forces of less than $1 \mathrm{meV} / \AA$ per atom, indicating that the optimised atomic locations are very close to the potential minimum. In addition, in contrast to the experiments, which obtain a structure at 
Table 1. Experimental and theoretical lattice parameters as used in this study. Experimental values are obtained from ref. [57]. All values quoted are in Angstrom.

\begin{tabular}{ccccccccc}
\hline \multicolumn{3}{c}{$\mathrm{a}$} & \multicolumn{3}{c}{$\mathrm{b}$} & \multicolumn{2}{c}{$\mathrm{c}$} \\
\hline PW91 & PBE & Experimental & PW91 & PBE & Experimental & PW91 & PBE & Experimental \\
\hline 9.565 & 9.550 & 9.693 & 9.683 & 9.755 & 9.524 & 7.737 & 7.727 & 7.537 \\
\hline
\end{tabular}

Table 2. Experimental and theoretical bond lengths. Experimental values are from reference [57]; again, all values are quoted in Angstrom.

\begin{tabular}{lccccc}
\hline & PW91 & PBE & PBE [49] & B3LYP/6-31+G(d) [67] & Experimental \\
\hline N1-C1 & 1.475 & 1.475 & & 1.476 & 1.475 \\
N1-H1 & 1.048 & 1.048 & & & 1.000 \\
N1-H2 & 1.050 & 1.051 & & & 0.861 \\
N1-H3 & 1.051 & 1.052 & & 1.509 & 0.950 \\
C1-C2 & 1.520 & 1.521 & & & 1.527 \\
C1-H4 & 1.089 & 1.093 & & 1.230 & 0.984 \\
C1-H5 & 1.089 & 1.088 & & 1.315 & 1.005 \\
C2-O1 & 1.206 & 1.207 & 1.237 & 1.444 & 1.223 \\
C2-N2 & 1.343 & 1.344 & & 0.850 & 1.338 \\
N2-C3 & 1.449 & 1.449 & & 1.525 & 1.455 \\
N2-H6 & 1.033 & 1.034 & & & 0.939 \\
C3-C4 & 1.513 & 1.513 & & & 1.524 \\
C3-H7 & 1.089 & 1.090 & & & 0.976 \\
C4-H8 & 1.091 & 1.091 & & 1.547 & 1.030 \\
C4-H9 & 1.087 & 1.089 & & 1.240 & 1.067 \\
C4-H10 & 1.088 & 1.089 & & 1.255 & 1.538 \\
C3-C5 & 1.538 & 1.539 & & & 1.249 \\
C5-O2 & 1.242 & 1.242 & 1.262 & & 1.256 \\
C5-O3 & 1.235 & 1.235 & 1.268 & &
\end{tabular}

room temperature, the DFT calculations are zero-temperature calculations; it is conceivable, therefore, that temperature effects may also explain at least some of the small discrepancy observed. Whilst such agreement is gratifying, it is likely that it is, to some extent, fortuitous. Semi-local XC functionals such as the GGA are incapable of treating van der Waals interactions; in molecular crystals, this may lead to inaccurate lattice constants [55]. It is likely that in this particular system, the zwitterionic nature of the glycyl-l-alanine molecule ensures that there is a significant electrostatic contribution to the intermolecular hydrogen bonds, and hence to the lattice energy, and thus the neglect of van der Waals interactions is not significant.

In Tables 2 and 3, the quality of the intra-molecular structures obtained are compared with the experimental values, through comparison of bond lengths and dihedral angles. The values in Table 2 indicate extremely good agreement with the experimental results for the intramolecular bond lengths. Note, however, that there is markedly less agreement for bond lengths involving hydrogen atoms. It is likely that this reflects the well-known difficulties of accurately locating hydrogen atoms via X-ray diffraction, as opposed to arising from a systematic problem in the theoretical treatment. The quality of the calculations compares favourably with other theoretical values found in the literature and quoted in Table 2. Optimisation of the crystal structure using the PBE functional [68] reveals that both functionals predict essentially identical bond lengths. This indicates, therefore, that the discrepancies that occur between our results and those of Zheng et al. [49] arise owing to numerical factors (choice of pseudopotential, kinetic energy cut-off, k-point sampling, or some combination thereof).

The calculated values of Kolev et al. [67] are obtained using DFT in conjunction with the B3LYP XC functional and the $6-31+\mathrm{G}(\mathrm{d})$ basis set. It is difficult to tell 


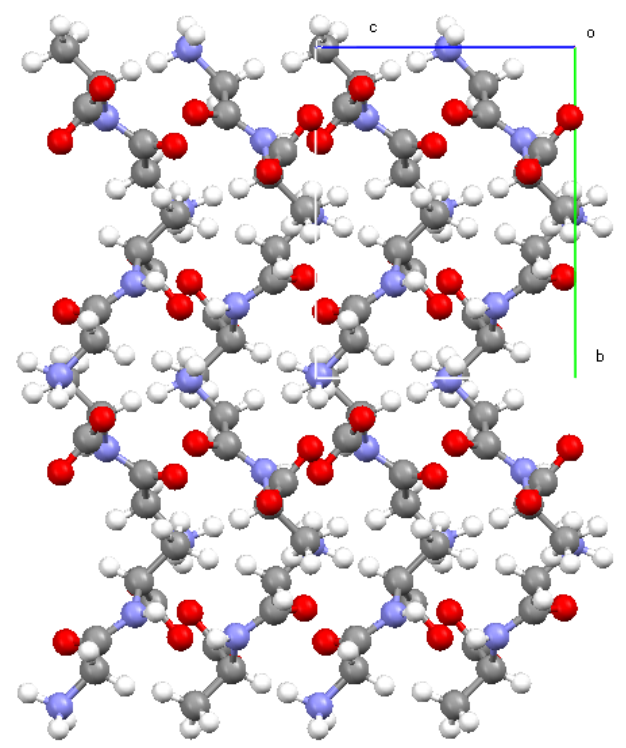

(a)

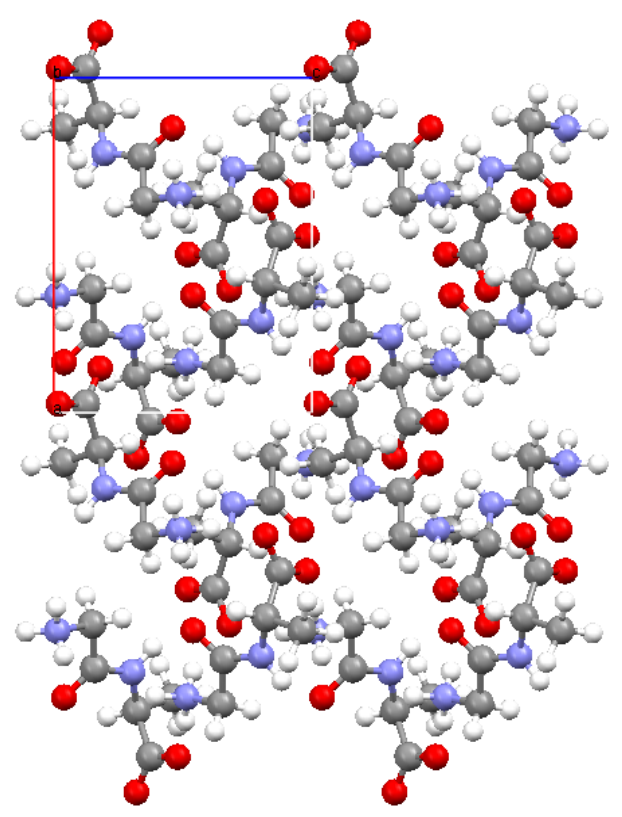

(b)

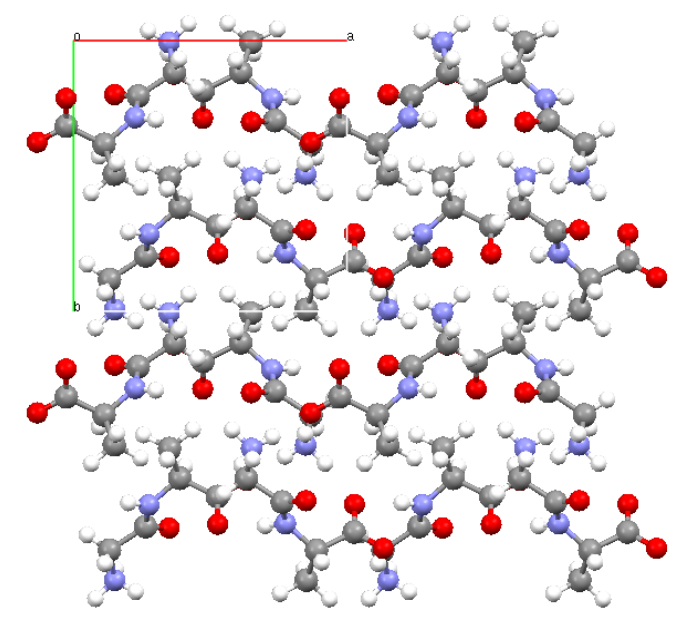

(c)

Figure 2. Crustal structure of the glycyl-1-alanine crystal. In each case a

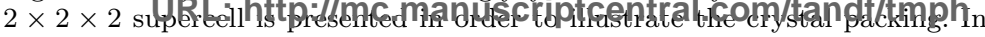
(a), the crystal is viewed along the $a$ axis; in (b), along the $b$ axis. In (c), it is viewed along the $c$ axis. 
Table 4. Selected intermolecular contacts - comparison between experiment (reference [57]) and theoretical values obtained in this work. All lengths are quoted in Angstrom angles quoted are in degrees.

\begin{tabular}{lcccc}
\hline & PW91 (this study) & PBE (this study) & B3LYP/6-31+G(d) $[67]$ & Experimental \\
\hline$\omega_{C^{\alpha}-C^{\prime}-N-C^{\alpha}}$ & -172.37 & -172.57 & 173.0 & -173.94 \\
$\phi_{C^{\prime}-N-C^{\alpha}-C^{\prime}}$ & -81.63 & -83.33 & -78.36 \\
$\psi_{N-C^{\alpha}-C^{\prime}-N}$ & -159.54 & -160.49 & -163.54 \\
\hline
\end{tabular}

\begin{tabular}{lccc}
\hline & PW91 (this study) & PBE (this study) & Experimental \\
\hline O2-H1 & 1.789 & 1.799 & 1.776 \\
O2-H2 & 1.724 & 1.723 & 1.923 \\
O3-H3 & 1.729 & 1.729 & 1.789 \\
O3-H6 & 1.881 & 1.891 & 1.969 \\
\hline
\end{tabular}

whether the discrepancies arise owing to differences in the functional used, or are basis-set artifacts; in addition, an ambiguity lies in these authors' work in that it is not clear as to whether their calculations refer to a gas-phase molecule, a periodic solid, or a cluster approximation to the crystal.

Having assessed the quality of the intra-molecular interactions through the bond lengths, in Table 3 we explore them further, through the determination of selected dihedrals. The calculated dihedrals are those commonly used to characterise the conformational preferences of peptides, namely $\phi, \psi$ and $\omega$. In common with the $\mathrm{X}$-ray data, the calculations indicate that the molecules are in the trans state. Agreement between experiment and theory for the dihedrals is poorer than that found for the bond lengths; similar results are found in gasphase calculations.

In Table 4, we examine the lengths of selected intermolecular contacts. It is noticeable that there is a variety of hydrogen bond lengths present, suggesting a hierarchy of hydrogen bond strengths; further, that each carboxylate oxygen atom can be seen to participate in two hydrogen bonds, i.e. it acts as a bifurcated acceptor. One of these bonds is an inter-layer bond, whilst the other is intra-layer in charatcer. The herring-bone-like packing of the molecules is such that hydrogen bonding involving the $\mathrm{O} 1$ atom does not occur. This is because the molecules pack so as to achieve the maximum number of interactions between the oppositelycharged amine and carboxy groups, i.e. it is interactions between these groups that drive the formation of the crystal structure. It is of interest to note that a recent examination of the structure of the aqueous solution using classical molecular dynamics [47] obtained a similar result, in which molecular aggregation was dominated by charge-charge interactions. Note that in some cases, disagreement between hydrogen bond lengths is marked. Some of this may be attributed to inaccuracies in the hydrogen positions as inferred from X-ray crystallography, whilst another contribution may be provided by the neglect of van der Waals interactions, as discussed earlier.

Summarising, therefore, it seems that intramolecular bonding interactions are well-described by the XC functional employed in this study, with discrepancies in the crystal structure arising in the main from differences in the treatment of intermolecular hydrogen bonds. Having discussed the structural features of the crystal in some depth, we now focus upon the electronic structure, and attempt to elucidate the relationship between the two. 
Figure 3. Electronic bandstructure of the glycyl-l-alanine crystal. This is a "zoomed-in" view, in which only the bands in the vicinity of the bandgap are displayed.

\section{Electronic Structure}

In Figure 3 we present the electronic bandstructure. Note that, owing to the presence of four molecules per unit cell, each molecular orbital splits into four bands. Examination of the bandstructure reveals that the crystal has three indirect bandgaps, which are found to arise from transitions between a conduction band minimum at $\mathrm{Z}$ and valence band maxima at the $\Gamma$-point, $\mathrm{Y}$ and $\mathrm{X}$. Although not corresponding to the true excitation energies, the Kohn-Sham eigenenergies may be taken as a zeroth order approximation to them [70]. Following this approach yields estimates for the indirect bandgaps of $4.95 \mathrm{eV}, 4.96 \mathrm{eV}$ and $4.95 \mathrm{eV}$, respectively. In comparison, the direct bandgap corresponding to $\Gamma-\Gamma$ transitions is $5.00 \mathrm{eV}$. As DFT calculations often obtain underestimates of the bandgap [71], this suggests that the glycyl-l-alanine crystal possesses an insulating character. These values are in close agreement with existing theoretical estimates of the bandgap for both crystalline alanine [55] and glycine [71]. In the absence of experimental data with which to compare directly, we note that experimental optical absorption measurements indicate a bandgap of $5.11 \mathrm{eV}$ for crystalline $\alpha$-glycine; it is perhaps possible to anticipate that the experimental value for glycyl-l-alanine is similar.

The valence bands displayed are extremely narrow, displaying little dispersion across the Brillouin zone, indicating that electronic orbitals are primarily localised on each molecule in the unit cell. Such localisation is consistent with hydrogen bonding being the dominant mechanism responsible for the crystal structure; similar findings have been reported concerning the electronic structure of amino acid molecular crystals [55, 71]. Inspection of the eigenvalues reveals that for the highest occupied valence bands the Davydov splitting (i.e. $E_{H O M O}-E_{H O M O-3}$ ) arising 
at the $\Gamma$-point is $0.20 \mathrm{eV}$; this is a relatively large splitting, and indeed is actually larger than the bandwidth arising from dispersion.

Interestingly, whilst the lowest conduction bands are similarly flat, the bands lying between 6 and $7 \mathrm{eV}$ show markedly more dispersion; this is characterised by a bandwidth of $0.70 \mathrm{eV}$. In particular, the dispersion is seen to be greatest along specific directions in reciprocal space, namely along the lines $\Gamma \rightarrow \mathrm{Z}, \mathrm{Y} \rightarrow \mathrm{S}$, $\mathrm{S} \rightarrow \mathrm{X}$ and $\mathrm{X} \rightarrow \mathrm{U}$. This can be understood by reference to the crystal unit cell as follows: the first direction mentioned corresponds to dispersion along the $c^{\star}$-axis; in real space, this is perpendicular to the $a b$ plane. This suggests that dispersion in this direction arises owing to the coupling of electronic states on neighbouring molecules, which is likely to be due to the O2-H1 hydrogen bonds, which are roughly directed perpendicular to this plane. Similar explanations may be offered for the other directions: the second direction corresponds to dispersion along the $b^{\star}$-axis, i.e. perpendicular to the $a c$ plane; this is found to be due to O3-H3 hydrogen bonds. The third direction in which marked dispersion occurs is along the $a^{\star}$-axis; this suggests that $\mathrm{O} 2-\mathrm{H} 2$ and O3-H6 hydrogen bonds are responsible for this. The magnitude of the Davydov splitting is also greater; at the $\Gamma$-point, this is $1.10 \mathrm{eV}$, compared to a splitting of approximately $0.2 \mathrm{eV}$ for the lowest lying conduction bands at the zone centre. Finally, marked dispersion occurs along the $c^{\star}$-axis, which as discussed earlier, corresponds to coupling occurring via O2-H1 hydrogen bonds.

Determination of the density of states, along with its decomposition in terms of angular momentum states, permits further insights into the electronic structure. To that end, in Figure 4, this quantity is shown. It is evident that the bands in the vicinity of the bandgap (both valence and conduction) are primarily of $p$-type character, whilst conduction bands between about 6 and $7 \mathrm{eV}$ are primarily of $s$-type character. The small amount of hybridisation that this indicates for the highest occupied states is consistent with the inference that the electronic orbitals are localised, as discussed earlier in the context of the bandstructure plot.

It is desirable to decompose these densities of states further, such that atomic contributions to the bandstructure may be obtained. In Figure 5, such local densities of states are presented for each atom type present in the crystal. It is apparent that the dominant contribution to the highest occupied valence states is provided by oxygen $p$-states. Further decomposition of this quantity (not shown) reveals that the carboxylate and carbonyl oxygens provide a comparable contribution to these states. This is interesting to note, especially if one recalls that the latter do not participate in hydrogen bonding, and it could therefore perhaps be anticipated that they do not contribute substantially to intermolecular interactions. It is also found that the carbonyl oxygen contributes marginally more to the LUMO states than the carboxylate oxygens. Nitrogen and carbon atoms each provide a contribution that is between approximately one sixth and one fifth that of the oxygen atoms to the HOMO states, with the nitrogen providing slightly more states. Decomposing the nitrogen local density of states into both amine and amide nitrogen contributions (not shown), it is found that the amine nitrogen makes a negligible contribution to the bandstructure in the vicinity of the bandgap, consistent with recent work concerning the electronic structure of the glycine molecular crystal [71]. In contrast to the amine nitrogen atom, the amide nitrogen atom is found to provide a more substantial contribution to the electronic states near the bandgap (not shown). In common with the oxygen atoms, it provides considerably more (by a factor of 2) to the valence band states than the conduction band states. Whilst the HOMO states are primarily $\mathrm{O} 2 p$ in character, the LUMO states are revealed to be an admixture of $p$ electrons from the oxygen, carbon and nitrogen atoms, with the dominant contribution arising from the carbons. 


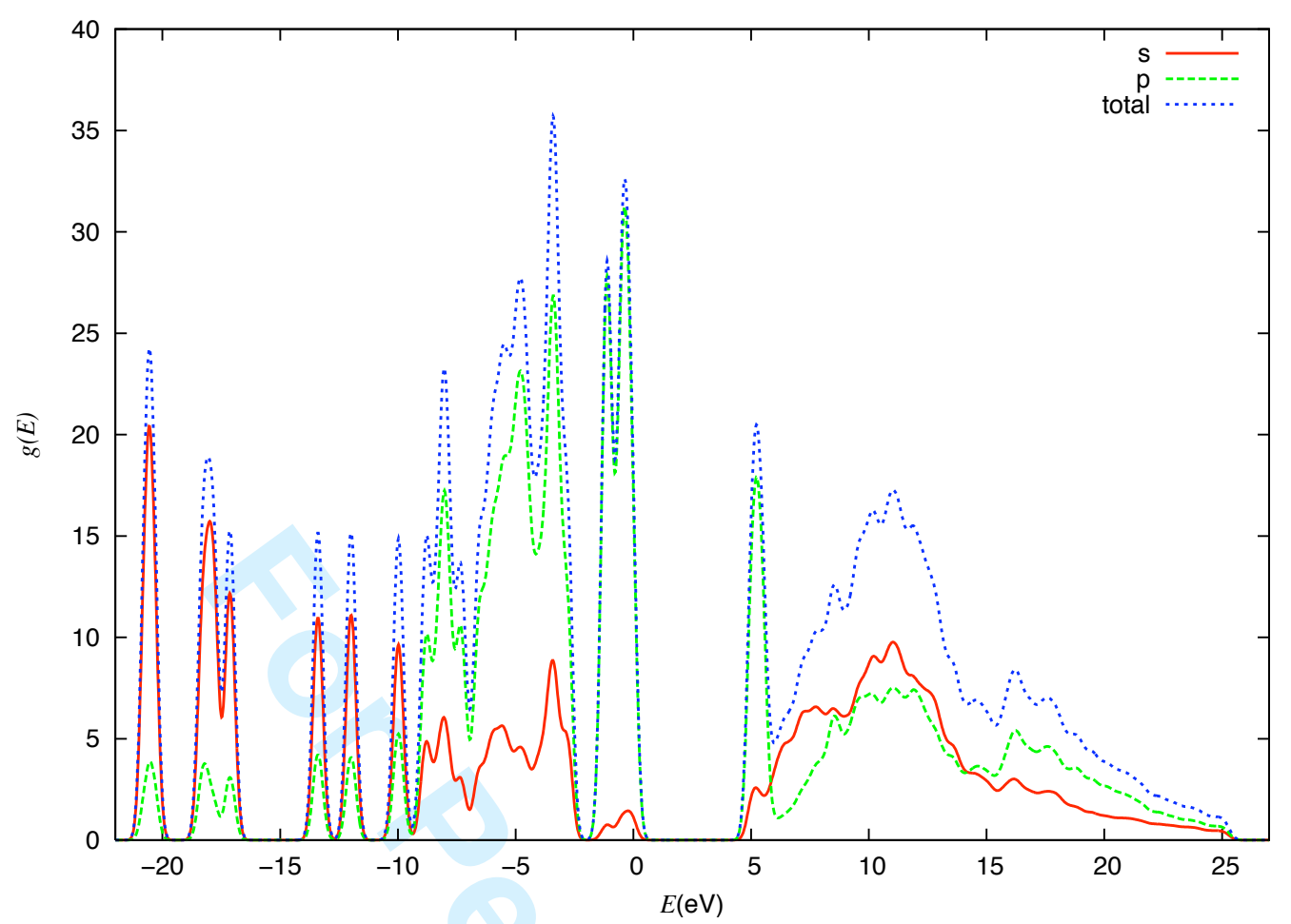

Figure 4. Density of states (DOS) for the glycyl-l-alanine crystal. Presented are both the total density of states and a decomposition in terms of angular momentum character. As in all of the DOS plots presented in this work, a Gaussian smearing of $0.2 \mathrm{eV}$ has been applied.

Taken together with the partial density of states, these plots indicate that the major contributions to the band of dispersive states between 6 and $7 \mathrm{eV}$ arise from carbon $p$ and hydrogen $s$-electrons. Figure 4 demonstrates that both types contribute appreciably, suggesting a marked degree of $s p$-hybridisation. This suggests that these states possess a more covalent character than the lowest lying states, which is consistent with the greater dispersion observed in the bandstructure.

\section{Lattice Dynamics}

Prior to discussing the zone-centre lattice dynamics, it is worthwhile to briefly elucidate the solid-state vibrational selection rules. The glycyl-l-alanine crystal possesses 237 normal modes (i.e. $3 \mathrm{~N}-3$, where 3 rigid translations must yield zero frequency by the acoustic sum rule); we note at this point that the degree to which the acoustic sum rule is satisfied provides some measure of the numerical precision and quality of the calculation. Our calculations satisfy this rule to within $20 \mathrm{~cm}^{-1}$, and indeed no imaginary frequencies are obtained, indicating that our calculated structure is mechanically stable and located close to the potential minimum. The factor group of the glycyl-l-alanine crystal is $D_{2}$; a factor group analysis therefore allows one to determine the transformation properties of the phonons. These are found to transform as

$$
\Gamma=60 a+59 b_{1}+59 b_{2}+59 b_{3}
$$

where the $a$ irrep is the only symmetry species that is not infra-red-active, and all symmetry species are Raman-active. Of these 237 modes, we shall concentrate 


\section{Molecular Physics}

Molecular Physics

1

4

5

9

10

11

12

13

14

15

16

17

18

19

20

21

22

23

24

25

26

27

28

29

30

31

32

33

34

35

36

37

38

39

40

41

42

43

44

45

46

47

48

49

50

51

52

53

54

55

56

57

58

59

60

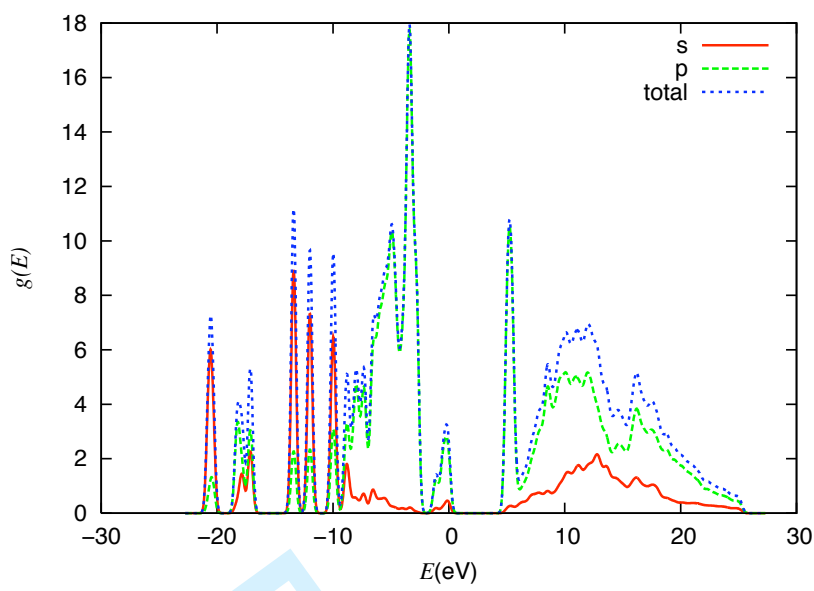

(a)

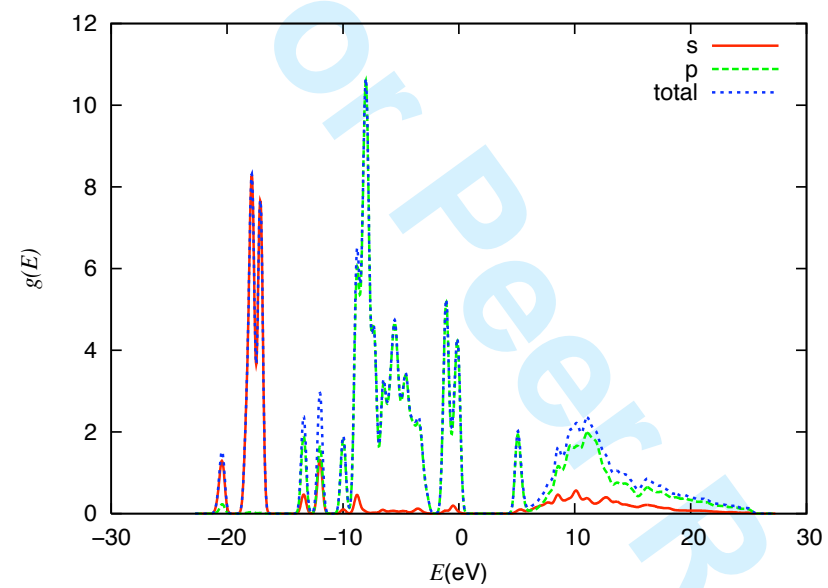

(c)

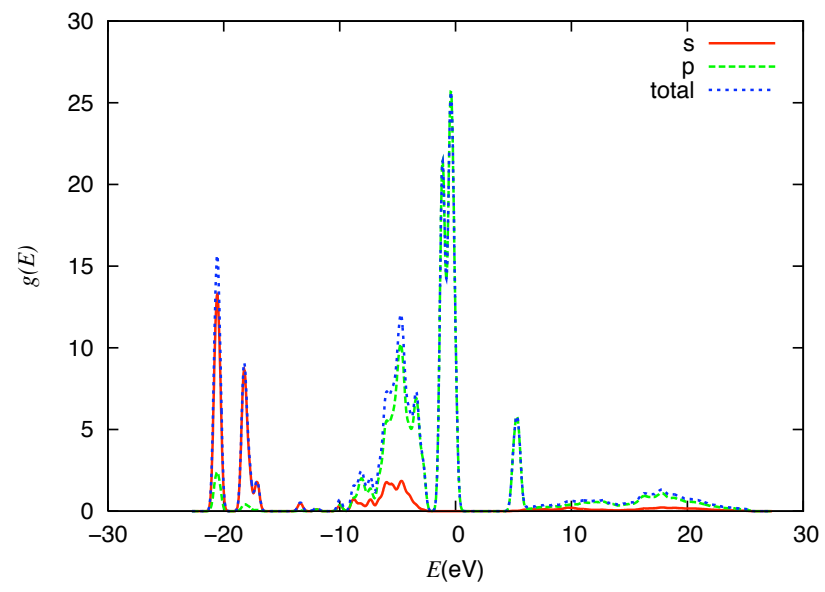

(b)

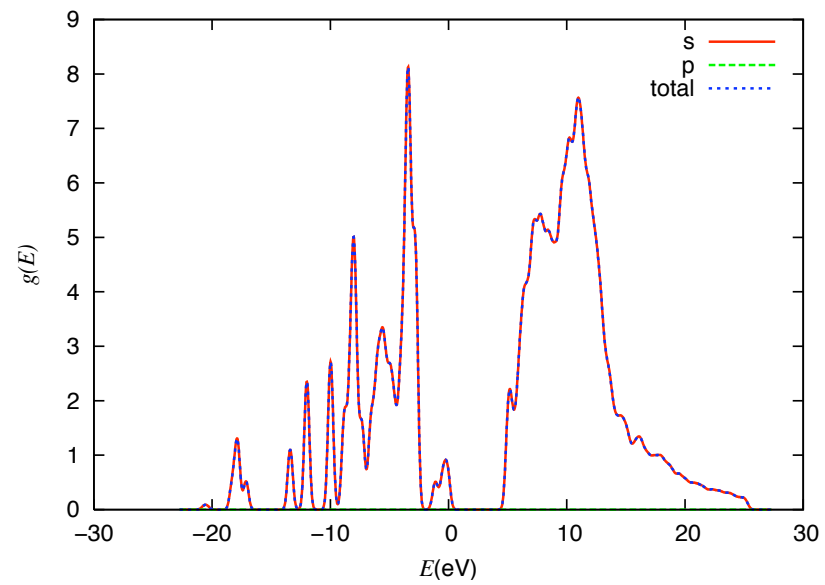

(d)

Figure 5. Local densities of states: in (a) for carbon atoms; in (b) for oxygen atoms; in (c) for nitrogen atoms; (d) hydrogen atoms.

our attention upon the vibronic modes. To motivate the discussion, in Figure 6, we present the calculated IR spectrum for the crystalline system. This is determined as described in [72]. Visualisation of the phonon eigenvectors corresponding to given mode frequencies allows the oscillations responsible for the IR features to be understood.

If we perform such an analysis and examine the IR spectrum, we initially address the major peaks. The large peak at around $3160 \mathrm{~cm}^{-1}$ corresponds to stretches of the amide $\mathrm{NH}$ bond. It is not entirely decoupled from other atomic motions, however; on the contrary, oscillations are also observed in the NH3 group. Furthermore, the NH3 oscillations are different in the different "layers" of the herring-bone structure; layers of alternating oscillators are formed, in which, in one layer, the NH3 group undergoes symmetric stretches, then in the next, undergoes asymmetric stretches, which appear almost like a "rotation" of the functional group. The presence of a peak at this frequency is found in the solid-state IR data presented by Kolev et al. [67]. It is interesting to note, however, that their calculations assign this oscillation a frequency of $3216 \mathrm{~cm}^{-1}$; it is not clear, though, whether their data refer to a crystal, or an isolated molecule. If it is the latter, then this would seem to be consistent with a red-shifting of the vibrational frequency upon crystallisation. Given that the amide group participates in a hydrogen bond with the carboxy group in the solid state, such a red-shift can be anticipated; however, compared 
Figure 6. IR spectrum of the glycyl-l-alanine crystal. A Gaussian smearing with of $20 \mathrm{~cm}^{-1}$ has been applied.

to the magnitude of red-shifts inferred from calculations on amino acids [56], the red-shift appears to be smaller than expected.

The second set of peaks of interest form a triplet at $\sim 2900 \mathrm{~cm}^{-1}, 2936 \mathrm{~cm}^{-1}$ and $2977 \mathrm{~cm}^{-1}$. The lowest frequency peak absorbs more strongly than the other two; this mode consists of asymmetric stretches in the terminal amine group. This peak forms a doublet with that at $2936 \mathrm{~cm}^{-1}$. This latter feature is revealed to arise from symmetric stretching of the bonds of the NH3 group in one layer, alternating with layers in which the bonds undergo a symmetric bending motion. It can perhaps be anticipated that such a motion will not result in as large a transition moment, explaining why this mode absorbs less strongly. The mode at $2997 \mathrm{~cm}^{-1}$ is somewhat similar, except that the symmetric stretches have instead been replaced by asymmetric stretches. We note that such modes do not excite any other molecular motions.

The other major absorption features are a doublet at $\sim 1547 \mathrm{~cm}^{-1}$ and 1566 $\mathrm{cm}^{-1}$. The lower frequency peak corresponds to a more complex collective motion, with the main motions arising in the carboxyl and amine groups, and lesser motions observed in the amide NH group. The carboxyl group motions are as follows: again, a "layer" structure is found, in which the carboxy groups in one layer undergo asymmetric stretches, with those in the next layer undergoing a rocking motion that is aligned along the molecular axis. The amine groups also undergo motion which is different in each layer: in one layer the NH bonds undergo a bending motion, in which all three bend in phase; in the next layer, the bonds undergo what appears to be a combination of bending and bonding, again, in phase. In the former case, the amide $\mathrm{NH}$ bond rocks along the molecular axis; in the latter, in contradistinction, the amide bond rocks perpendicular to the molecular axis. These differences in 


\section{Page 13 of $15 \quad$ Molecular Physics}

Molecular Physics

Table 5. Optical $\left(\epsilon^{\infty}\right)$ and static $\left(\epsilon_{0}\right)$ contributions to the dielectric permittivity.

\begin{tabular}{lccccc}
\hline \multicolumn{3}{c}{$\epsilon^{\infty}$} & & \multicolumn{3}{c}{$\epsilon_{0}$} \\
\hline 2.37 & 0.00 & 0.00 & 3.59 & 0.00 & 0.00 \\
0.00 & 2.31 & 0.00 & 0.00 & 4.64 & 0.00 \\
0.00 & 0.00 & 2.32 & 0.00 & 0.00 & 3.71 \\
\hline
\end{tabular}

the motion between layers may be understood in terms of the hydrogen bonding network present in the crystal; for example, in cases where the amide $\mathrm{NH}$ oscillates parallel to the molecular axis, then this may correlated with the carboxy group rocking; conversely, $\mathrm{NH}$ rocking perpendicular to the molecular axis correlates with asymmetric stretches of the carboxy group. That is, the carboxy and amide group oscillations are coupled through the intermolcular hydrogen bonds present in the crystal. This demonstrates that, even for high frequency intramolecular modes, intermolecular interactions play an important part in determining the nature of the oscillations. Thus, the effect of intermolecular interactions is not simply to cause a shift in the frequency of intramolecular vibrations; rather, they may also couple intramolecular vibrations on neighbouring molecules. We note briefly that the oscillations leading to the higher frequency peak are the same; however, the amplitude is increased, which explains the increased size of the absorption peak at this frequency.

The smaller peak at $\sim 1660 \mathrm{~cm}^{-1}$ arises from pronounced bending of the $\mathrm{NH}$ bonds within the amide group that are almost entirely decoupled from any other atomic motions. At $\sim 1714 \mathrm{~cm}^{-1}$, we find the amide I bands, arising from stretching of the amide CO bonds. Given that this involves backbone atoms, in contrast to the above excitations, we find a collective motion in which other atoms are also induced to move; for example, some rocking of the amide $\mathrm{NH}$ bonds is found, in which alternaing layers execute this motion either parallel to, or perpendicular to, the molecular axis. As above, these differences are correlated with differences in the carboxy group oscillations. Bending of the $\mathrm{NH}$ bonds in the amine group are also found.

Finally, in Table 5, we present both the optical permittivity, $\epsilon^{\infty}$, and the static dielectric permittivity, $\epsilon_{0}$. It is noticeable that the static (i.e. ionic) contribution to the permittivity is greater than the optical (i.e. electronic) contribution. This result can be anticipated for the following reason: both the IR absorption and the static permittivity are proportional to the oscillator strength; therefore, significant IR absorption, as observed above, implies a large non-zero oscillator strength. This will then manifest itself in the static contribution to the permittivity.

\section{Conclusions}

In this work we have examined, in detail, the structural, electronic and lattice dynamical properties of the glycyl-l-alanine molecular crystal using density functional theory. Our calculations reveal that the crystal is bound together by a hydrogen bond network, with molecules being arranged within the unit cell to maximise the number of hydrogen bonds possible between the oppositely-charged amine and carboxy functional groups. Indeed, the calculations reveal that a hierarchy of hydrogen bond strengths exists within the crystal, with those between amide and carboxy oxygens being weaker than those between oppositely-charge functional groups. Recent studies of organic molecular crystals [55] have suggested the importance of adequately treating van der Waals interactions, with a failure to do so manifesting itself in overestimates of lattice constants. In contrast, in this work 
the theoretically-determined lattice constants are found to be within $2 \%$ of the experimental values, suggesting that hydrogen bonds are dominant in stabilising the crystal, and that the neglect of van der Waals interactions implicit in the GGA $\mathrm{XC}$ functionals employed in this study is not significant. The slight overestimation of the $b$ and $c$ axes relative to experiment is consistent with the known behaviour of GGA functionals (i.e. they tend to underbind); in contrast, the underestimate of the $a$ axis suggests that, in this case, the GGA may over-bind.

Bandstructure calculations indicate that the glycyl-l-alanine crystal is a wideindirect-gap insulator, with indirect bandgaps of $4.95 \mathrm{eV}$, as compared to a direct bandgap of $5.00 \mathrm{eV}$. The details of the bandstructure, namely, the directional dependencies of the bandwidths are found to be directly correlated with the hydrogen bond orientations in the unit cell, suggesting that this, and by extension, the molecular arrangement, plays an important role in determining the bandstructure, and that it is coupling of electronic states via these hydrogen bonds that results in band dispersion. Dispersion is found to be particularly prevalent in the conduction band states; the valence band states show little dispersion. This is consistent with localised molecular orbitals and primary intermolecular interactions via hydrogen bonds; it is further supported by the density of states plots, which indicate that these states primarily arise from oxygen $2 p$-states, and indicate a lack of hybridisation. In contrast, the dispersive conduction band states are found to possess a more pronounced hybridisation between hydrogen $s$ - and carbon $p$-states.

The lattice dynamical and IR absorption calculations reveal that major absorption features arise owing to excitation of specific functional groups and/or bonds whereas, in contrast, the weak absorption features rather arise from motions of the molecular backbone, and hence couple several functional groups resulting in motions that are more difficult to characterise. However, even in the intramolecular modes, the presence of intermolecular hydrogen bonds is found to couple intramolecular motions in neighbouring molecules, demonstrating that an accurate treatment of intermolecular forces is necessary to obtain accurate intramolecular modes.

It is hoped that these calculations will permit greater insight into the electronic and lattice dynamical properties of peptidic molecular crystals, and that these may yield insights into the structural motifs observed in liquid-state peptides. More particularly, molecular crystal calculations allow, through crystal polymorphism, the effects of varying inter- and intramolecular interactions and molecular conformation upon electronic properties to be understood. Understanding this relationship potentially opens up avenues by which electronic and optical properties may be modulated by mechanical (conformational) changes in the molecular structure, which is of interest to technological exploitation of such molecules. We aim, therefore, to use this work as a foundation to investigate and elucidate this relationship.

\section{Acknowledgements}

The authors would like to acknowledge the UK HECToR supercomputing facility, which was used to perform these calculations.

\section{References}

[1]H. Imamura and M. Kato, Proteins - Structure Function and Bioinformatics 75 (4), 914 (2009).

[2] L.L. Lee, H. Ha, Y.T Chang et al., Protein Science 18 (2), 277 (2009).

[3]B. Fierz and T. Kiefhaber, J. Am. Chem. Soc. 12 (3), 672 (2007).

[4] N.S. Hinrichs and V.S. Pande, J. Chem. Phys. 126 (24), 244101 (2007). 
[5]F. Noe and S. Fischer, Curr. Op. Struct. Biol. 18 (2), 154 (2008).

[6]Y. Suzuki, J.K. Noel and J.N. Onuchic, J. Chem. Phys. 128 (2), 025101 (2008).

[7]P.R. Tulip, R.Z. Troitzsch, E. Cerasoli et al., in preparation (2009).

[8]S. Kannan and M. Zacharias, J. Struct. Biol. 166 (3), 288 (2009).

[9] M. Khalili and D.J. Wales, J. Chem. Theor. Comput. 5 (5), 1380 (2009).

[10]S. Hofinger, B. Almeida, and UHE Hansmann, Proteins - Structure Function and Bioinformatics 68 (3), $662(2007)$.

[11]P. Campiglia, C. Esposito, M. Scrima, et al., Chem. Biol. Drug Des. 69 (2), 111 (2007).

[12] C. Soto, R.J. Kascsak, G.P. Saborio, et al., The Lancet 355 (9199), 192 (2000).

[13]D.J. Gordon, R. Tappe and S.C. Meredith, J. Peptide Res. 60 (1), 37 (2002).

[14]L. Bennett, R. Williams, H. Ecroyd et al., Aust. J. Dairy Tech. 60 (1), 117 (2009).

[15]C. Nerelius, J. Johansson and A. Sandregen, Front. Bioscience 14, 1716 (2009).

[16]S.Y. Chung, S. Lee, C. Liu et al., J. Phys. Chem. B 113 (1), 292 (2009).

[17]T. Tabarin, A. Kulesza, R. Antoine et al., Phys. Rev. Lett. 101 (21), 213001 (2008).

[18]R. Mitric, J. Petersen, A. Kulesza et al., J. Chem. Phys. 127 (13), 134301 (2007).

[19]R. Mitric, J. Petersen, A. Kulesza et al., Chem. Phys. 343 (2-3), 372 (2008).

[20]A. Kulesza, R. Mitric and V. Bonacic-Koutecky, J. Phys. Chem. A 113 (16), 3783 (2009).

[21]E.M. Krauland, B.R. Peelle, K.D. Wittrup et al., Biotech. Bioeng. 97 (5), 1009 (2007).

[22] J.E. Zull, J. Reedmundell, Y.W Lee et al., J. Indust. Micrbiol. 13 (3), 137 (1994).

[23] M. Mijajlovic and M.J. Biggs, J. Phys. Chem. C 111 (43), 15839 (2007).

[24]K.E. Sapsford, Y.S. Shubin, J.B. Delehanty et al., J. Appl. Microbiol. 96, 47 (2004).

[25]M. Sarikaya, C. Tamerler, A.K.Y. Jen et al., Nat. Mater. 2, 577 (2003).

[26]E. Katz and I. Willner, Angew. Chem. Int. Ed. 43, 6042 (2004).

[27] C.M. Niemeyer, Angew. Chem. Int. Ed. 40, 4128 (2001).

[28] S.V. Patwardhan, G. Patwardhan and C.C. Perry, J. Mat. Chem 17, 2875 (2007).

[29]E.E. Oren, C. Tamerler and M. Sarikaya, Nano Lett. 5 (3), 415 (2005).

[30] L.M. Ghiringelli, B. Hess, N.F.A. van der Vegt et al., J. Am. Chem. Soc. 130, 13460 (2008).

[31]S. Monti, V. Carravetta, C. Battocchio et al., Langmuir 24 (7), 3205 (2008).

[32] R.Z. Troitzsch, H. Vass, W.J. Hossack et al., J. Phys. Chem. B 112 (14), 4290 (2007).

[33]S. Köppen, O. Bronkalla and W. Langel, J. Phys. Chem. C 112, 13600 (2008).

[34]A.A. Mungikar and D. Forciniti, Biomacromolecules 5 (6), 2147 (2004).

[35] P. Hohenberg and W. Kohn, Phys. Rev. 136 (3B), B864 (1964).

[36]W. Kohn and L.J. Sham, Phys. Rev. 140 (4A), 1133 (1965).

[37] M.C. Payne, M.P. Teter, D.C. Allan et al., Rev. Mod. Phys. 64 (4), 1045 (1992).

[38]R.Z. Troitzsch, P.R. Tulip, J. Crain et al., Biophys. J. 95 (11), 5014 (2008).

[39] C.J. Pickard and R.J. Needs, Phys. Rev. Lett. 102 (12), 125702 (2009).

[40] V. Thakor, J.B. Staunton, J. Poulter et al., Phys. Rev. B 68 (13), 134412 (2003).

[41]R.M. Martin, Electronic Structure: Basic Theory and Practical Methods (Cambridge University Press, 2004).

[42] G. Nandini and D.N. Sathyanarayana, J. Mol. Struct. Theochem. 638, 79 (2003).

[43]R. Jacob and G. Fischer, J. Mol. Struct. 613 (1-3), 175 (2002).

[44]T. Liang and T.R. Walsh, Mol. Sim. 33 (4-5), 337 (2007).

[45] H.W. Hugosson, A. Laio, P. Maurer et al., J. Comput. Chem. 27 (5), 672 (2006).

[46]S.E. McLain, A.K. Soper, I. Diadone et al., Ang. Chem. Int. Ed. 47, 9059 (2008).

[47]P.R. Tulip and S.P. Bates, J. Chem. Phys. 131, 015103 (2009).

[48]Y. Wei, D-K. Lee and A. Ramamoorthy, J. Am. Chem. Soc. 123, 6118 (2001).

[49] A. Zheng, S-B. Liu and F. Deng, J. Comput. Chem. 30, 222 (2009).

[50]R. O. Jones and O. Gunnarsson, Rev. Mod. Phys. 61 (3), 689 (1989).

[51]S.J. Clark, M.D. Segall, C.J. Pickard et al., Zeit. Krist. 220 (5-6), 567 (2005).

[52]L. Kleinman, D. M. Bylander, Phys. Rev. Letters 48, 1425 (1982).

[53] H.J. Monkhorst and J.D. Pack, Phys. Rev. B. 13 (2), 5188 (1976).

[54] J.P. Perdew and Y. Wang, Phys. Rev. B 46, 12947 (1992).

[55]P.R. Tulip and S.J. Clark, Phys. Rev. B 71 (19), 195117 (2005).

[56]P.R. Tulip and S.J. Clark, Phys. Rev. B 74 (6), 064301 (2006).

[57] A.H.J. Wang and I.C. Paul, Cryst. Struct. Comm. 8 (2), 269 (1979).

[58] S.L. Price, Cryst. Eng. Comm. 6, 344 (2004).

[59] C. Ouvrard and S.L. Price, Cryst. Growth Des. 4, 1119 (2004).

[60] M.A. Neumann, F.J.J. Lensen and J. Kendrick, Angew. Chem. Int. Ed. 47, 2427 (2008).

[61]X. Gonze and C. Lee, Phys. Rev. B 55 (16), 10355 (1997).

[62]K. Refson, P.R. Tulip and S.J. Clark, Phys. Rev. B 73 (15), 155114 (2006).

[63]P.R. Tulip and S.J. Clark, J. Chem. Phys. 121 (11), 5201 (2004).

[64]B. Winkler, J.D. Gale, K. Refson et al., Phys. Chem. Minerals 35 (1), 25 (2008).

[65] G.K. Horton and A.A. Maradudin, Dynamical Properties of Solids Vol 1 (North-Holland Publishing Company, Amsterdam 1974).

[66]P.U. Jepsen and S.J. Clark, Chem. Phys. Lett. 442 (4-6), 145 (2007).

[67]Ts. Kolev, B.B. Koleva, S.Y. Zareva et al., Inorg. Chim. Acta 359, 4367 (2006).

[68] J.P. Perdew, K. Burke and M. Ernzerhof, Phys. Rev. Lett. 77, 3865 (1996).

[69]R.S. Mulliken, J. Chem. Phys., 23 (10), 1833 (1955).

[70] A. Seidl, A. Görling, P. Vogl et al., Phys. Rev. B 53 (7), 3764 (1996).

[71]M.Z.S. Flores, V.N. Freire, R.P. dos Santos et al., Phys. Rev. B 77 (11), 115104 (2008).

[72]P.R. Tulip, Ph.D. thesis, University of Durham, 2004. <http:/ / portellen.phycmt.dur.ac.uk/sjc/> 